\title{
A review on health evaluation methods of domestic urban ecosystem
}

\author{
L.P. FENG \& H.B. JIN \& Y.D. JIANG \\ Business Administration School, HoHai University, China \\ * Lan-ping Feng
}

KEYWORD: Urban ecosystem; Health evaluation; Evaluation methodology

ABSTRACT: By carding the researches of health evaluation methods of domestic urban ecosystem, this paper discusses the principles, methods and revealed problems of urban ecosystem health evaluation, in order to providing reference for the building and evaluation of urban ecosystem health evaluation system.

\section{introduction}

As people pay more attention to the ecological environment, the research on the ecosystem health is getting more and more deeply, the health evaluation of the urban ecosystem is an important developing direction of the ecosystem health research. The health of urban ecosystem refers to the health of urban living environment, a continuous virtuous cycle formed by the material and energy exchanging between the environment and the community in the system human's manufacture and life, and the health of human populations in urban ecosystem. Along with the rapid development of urbanization in China, a series of problems of urban ecological environment are brought out, such as serious environmental pollution, ecological restoration, and ecological selfpurification ability and so on. How to evaluate the health of urban ecosystem becomes an important research subject.

This paper carries out statistics on the document of the field of urban ecosystem health evaluation in the full text database of the 2003-2014 annual periodicals, as shown in table1.

Table 1: 2003-2004 Document quantity of urban ecosystem health evaluation

\begin{tabular}{|c|c|c|c|c|c|c|}
\hline Year & 2003 & 2004 & 2005 & 2006 & 2007 & 2008 \\
\hline Quantity & 3 & 1 & 13 & 19 & 17 & 22 \\
\hline Year & 2009 & 2010 & 2011 & 2012 & 2013 & 2014 \\
\hline Quantity & 19 & 15 & 17 & 14 & 13 & 15 \\
\hline
\end{tabular}

The time distribution of the academic papers reflects the development of the academic research in this field. There are only 4 paper from 2002 to 2004, and it is in the initial stage. By 2005 it reached 13, the growth rate is larger. From 2006, the studies have steadily increasing year by year, and there are some minor fluctuations.

The key of scientific evaluation, forecasting and decision-making of urban ecosystem health is to select the evaluation index and build the evaluation model. Through the analysis of the relevant literature, domestic researches on the health evaluation of urban ecosystem mainly focus on the theory, the selection and the construction of the evaluation index, research and analysis of the health of the individual city ecosystem or a comparative study on the health of multiple urban ecosystems. 
By carding the researches of methods of domestic urban ecosystem health evaluation, this paper discusses the principles, methods and revealed problems of urban ecosystem health evaluation, in order to provide scientific basis for the healthy development of urban ecosystem and the sustainable development of urban ecological environment and economic society.

\section{Health of urban ecosystem}

From the beginning of the 80's in twentieth Century, the ecosystem health science has become the cross and integrated science of natural science, social science and human health science. The measurement of the health of the ecosystem has caused wide attention of many scholars since Schaeffer et al. raised it. With regard to the concept of ecosystem health, Rapport et al. concluded that "The status, condition or performance of an ecosystem defined by the appropriate target as the standard." That is, the health of the ecosystem should include the connotation of 2 aspects: the ability to meet the reasonable demands of human society and the ability of the ecosystem to maintain itself and renew itself. The urban ecosystem is different from the natural ecosystem, it is disturbed by human activity, has evolved into highly artificial natural, social, economic compound ecosystem. Qin Qu et al. believed that the health of the urban ecosystem refers to the ecological system, and it is composed of natural, economic and social, the energy flow and material cycling between the inner productive life and the environment is not destroyed. Qiu Tan and Deng Zhaolong believe that the urban ecosystem is the core of the human production activity, and the city is added two subsystems of the society and economy based on the natural ecosystem. Urban ecosystem is a complex ecosystem of society, economy and nature. Yu Ya-juan et al. think that the urban ecosystem is a complex ecosystem which is composed of 5 subsystems which are interrelated and have their own characteristics, such as bearing capacity, support force, attraction force and continuation force, vibration force etc. Yu Hong -lu, Li Fu-ping believes that the urban ecosystem belongs to artificial ecosystem. Chen Cheng and Shi Huichun considered that the objective of urban ecosystem health evaluation was to diagnose the damage or degradation degree caused by natural factor and human activity, in order to provide the target basis for the managers and decision-makers, so that the urban ecosystem can be better utilized, protected and managed.

\section{Construction of index system}

The health evaluation of the urban ecosystem is an important part of the health evaluation of the ecosystem. Different scholars have different views on construction of the evaluation index system. At present, many domestic scholars generally view, referring to Rapport's ecosystem health index of natural ecosystem, is to construct health evaluation index according to the particularity, vigor, organizational structure, resilience, ecosystem services and human health of the urban ecosystem. Based on the concept that city, dominated by artificial, the complex ecosystem of nature, economy, society, Yan Wen-tao, Zhang Mei-zhu, etc. construct the health evaluation index system of urban ecosystem in three aspects: natural subsystem, social subsystem and economic subsystem, and construct the urban ecosystem health index system from the perspective of the concept and influence factor of ecosystem health. Yu Ya-juan, et al based on the compound ecosystem theory, from the city CSAED model to build the urban ecosystem health evaluation model. Su Meirong introduces the concept of urban life, and constructs urban vitality index, which includes the productivity, life state, ecological potential and vitality, She reflects the health status of urban ecosystem comprehensively according to the economic subsystem, the social subsystem, the natural subsystem and the ecological subsystem respectively. Qin Qu et al. establish the urban ecosystem 
health evaluation index system from 3 aspects of structure function, sustainable utilization ability and dynamic change. It use the entropy method to calculate the weight of each index value, apply grey correlation analysis theory to construct the health evaluation model of urban ecosystem, and conduct a case study of 9 cities in Guizhou Province. From the connotation of urban ecosystem health, Ceng Yong constructs the framework of urban ecosystem health index system by using pressure, state and response mechanism .

The core of urban ecosystem health evaluation is the construction of evaluation index system, and there is no consensus at this point. Due to the differences in understanding the health concept of urban ecosystem and the different points of evaluation, the different evaluation index system is proposed. When it comes to the selection of each specific index, some of the scientific nature of the indicators need to be discussed. Such as, the indexes of the evaluation are too many, the natural, the environment, the resources, the ecology, the economy, the society and other factors all contained. It is difficult to reflect the essence and the connotation of the city ecosystem health; Some indexes seem reasonable, but there are some problems such as data is difficult to obtain, operation difficulty and so on, and some indicators also exist cross repeat phenomenon. In view of the fact that the index system can reflect the ecological system health requirements in many aspects. Therefore, more commonly used evaluation method is selecting suitable and representative index system, reflecting the status of ecosystem health by evaluating the index system.

\section{Health evaluation of urban ecosystem}

Its evaluation method mainly include: fuzzy mathematical method, projection pursuit method, grey system evaluation method, comprehensive attribute model evaluation methods etc.

\section{Fuzzy comprehensive evaluation method}

In recent years, along with the application of mathematical model method and its ideas in ecology and geography, and considering a problem that the urban ecosystem is healthy or not has some certain fuzziness, many scholars try to use the mathematical model to evaluate the health status of the urban ecosystem quantitatively, and get more research results in this field. Fuzzy comprehensive evaluation method is making a scientific evaluation on the quality of things based on the comprehensive consideration of various factors affecting something, by applying fuzzy mathematics and fuzzy statistical method. For example, GUO Xiu-rui, Wei Fei-yang etc, Wen Qi, Ying Hua Zheng etc, used fuzzy evaluation method to carry on the empirical research respectively of Guangzhou, Nanchong, Yinchuan, Foshan and other cities. Li Shuang-jiang, Luo Xiao and Hu Ya-ni used the principle and method of fuzzy comprehensive evaluation to study the health of the ecosystem in Shijiazhuang from 2005 to 2009, and its result can mostly reflect the development trend of the ecosystem health status. Chen Kelong used the method of fuzzy mathematics evaluation to evaluate and analyze urban ecosystem health status of Xining from 2000to 2008.

\section{Projection pursuit method}

The projection pursuit method first appeared in twentieth Century 60 at the end of the 1990s, Kruscal gel first used projection pursuit method to project high-dimensional data onto a low dimensional space, by calculating, maximized the index that reflecting data aggregation degree, to find the optimal projection direction reflecting data structure features. The system has many variables and the weights are difficult to determine, projection pursuit model is introduced to carry out a case study of the health status of urban ecosystem. According to the size of the best projection component of each index, projection pursuit method makes it clear of the important index that has the impact on the evaluation and classification of urban ecological health, to provide a reference for 
improving the ecological function and construction of cities in the future. For example, CHEN Guang-Zhou, WANG Jia-Quan used a new type of multi index data processing method--projection pursuit, Selected the typical index, found the best projection direction by the genetic algorithm optimization based on real number coding, evaluated the health status of the urban ecosystem in 2000 and 2005 in Guangzhou. Yu su-jian selected the typical index by projection pursuit method, found the best projection direction by the genetic algorithm optimization based on real number coding, and evaluated the health status of the urban ecosystem of Fuzhou, Xiamen and Quanzhou in 2007.

Besides, the model can accurately depict the specific health level of the ecosystem and has a higher evaluation accuracy and the district degree compared with the fuzzy comprehensive evaluation method.

\section{grey correlation degree method}

The grey correlation analysis belongs to the category of geometric processing from the perspective of method, essentially, it is the geometric comparison of the data sequence which reflects the change of the factors. It is used to analyze the grey degree of the correlation degree between the measurement factors. In addition, the conclusion can be get through the comparison of the correlation curves between the various factors. If the similarity degree of sequence curves geometry is close, the correlation degree between the corresponding sequences is larger, and the vice versa is smaller. The urban ecosystem is a highly artificial compound ecosystem, in the longterm development, the function and relationship of the elements of the city ecosystem and other elements experienced the process from black to grey. Therefore, the urban ecosystem has the typical grey system character, grey correlation analysis can be applied to compare the correlation degree of the optimal vector of each index, and the health status of urban ecosystem is evaluated. Applying grey forecasting model to evaluate the health of urban ecosystem can get more objective and comprehensive evaluation results. For example, Qin Qu, Dai Wen, Zhang Yong-rong used grey correlation analysis theory to build a urban ecosystem health evaluation model, and carried out a case study of 9 cities in Guizhou Province. Guo Ruili calculated the weight coefficient of indexes by using the information entropy to reflect the utility value of the data itself, and established a entropy weight comprehensive evaluation model to evaluate the health of urban ecosystem in Chongqing. Finally, according to the results of the evaluation and the grey theory, the grey prediction model of GM (I, I) is established to predict the ecosystem health of Chongqing city. Model evaluation method of attribute synthetic model

Attribute comprehensive model is a kind of evaluation model, which is based on the analysis of attribute set and attribute measure, and is a quantitative study of the qualitative description of things or natural phenomenon. It is composed of three parts, single index attribute measure analysis, comprehensive attribute measure analysis, attribute recognition and score criterion. The key of the evaluation method is that if the index system established could reflect the attribute of the thing (or phenomenon) that is studied, that is, the determination of attribute function and evaluation index weights. For example, Yan Wen-tao used the model and method to evaluate the health status of the new ecosystem in northern Chongqing. Zhang Meizhu constructed the evaluation model according to the attribute theory, and evaluated the health of the urban ecosystem in Liupanshui.

The health evaluation of urban ecosystem is not only of the above research methods, but also the set pair analysis method and so on. These methods are seldom studied in the health evaluation of urban ecosystem, so these methods are not introduced alone. 


\section{Existing problems}

The existing evaluation method is very good for the health of urban ecosystem, but there are some problems and common defects in the practical evaluation. For example the quantitative standard of the evaluation indexes is inaccurate, and there is no generally accepted standards about quantitative evaluation standard, and how to determine the index weight reasonably and scientifically is also a problem worthy of further study. What's more, in the current study, a certain evaluation method is mainly used, and the different evaluation methods are seldom applied to the same sample.

\section{Acknowledgment}

This work is partially supported by General Research for Humanities and Social Sciences Project, and Chinese Ministry of Education(Grant No. 11YJA870007), and the Fundamental Research Funds for the Central Universities(Grant No. 2013B09314).

\section{References}

[1] Chen Cheng; Shi Hui-chun. 2013. Health Evaluation on Urban Ecosystem of Baiyin City. Journal of Gansu Agricultural University48(4): 94-99.

[2] Chen Guang-Zhou, Wang Jia-Quan. 2009. Health Assessment of Urban Ecosystem Based on a Projection Pursuitmodel. Acta Ecologica Sinica29(9): 4918-4923.

[3] Chen Ke-long, Su Mao-xin, Li Shuang-cheng, Lu Jing-hua, Chen Ying-yu, Zhang Fei, Liu Zhijie. 2010. The Health Assessment of the Urban Ecosystem of Xining City. Geographical Research 29(2): 214-222.

[4] Guo Rui-li, Zheng Qin-yu, Liu Juan, Li Mei-rong.2012. Ecosystem Health Assessment of Chongqing City Based on Entropy and GM $(1,1)$ Model. China Environmental Science32(6): 11481152 .

[5] Guo Xiu-rui, Yang Ju-rong, Mao Xian-qiang. 2002. Primary Studies on Urban Ecosystem Health Assessment. China Environmental Science22(6): 525 529.

[6] Li Shuang-jiang, Luo Xiao, Hu ya-ni. 2012. Ecological Health Assessment of Shijiazhang Urban Ecosystem in Fasting Urbanizing City. Research of Soil and Water Conservation 19(3): 245249.

[7] Lu Yu-hong, Li Fu-ping. 2008. Health Assessment and Case Analysis of Urban Ecosystem. Environmental Protection(18): 39-41.

[8] Qin Qu, Dai Wen, Chen Zhi-xia. 2014. Review of researches on the evaluation of urban ecosystem health in china. Safety and Environmental Engineering21(4): 96-100, 106.

[9] Qin Qu, Dai Wen, Zang Yong-rong. 2014. Assessment of Urban Ecosystem Health Based on Gray Correlation Analysis. Journal of Anhui University(Natural Science Edition)(4): 102-108. [10] Qiu Tan, Liu Zhao-long. 2014. Health Assessment and Comparative Analysis of Urban Ecosystem in Beijing--Empirical Analysis Based on Beijing and Shenzhen. Urban Geography(22): 159-161.

[11] Su Mei-rong, Yang Zhi-feng, CHEN Bin. 2010. Urban Ecosystem Health Assessment Based on Vitality Index and Set Pair Analysis. China Population Resources and Environment20(2): 122128.

[12] Wen Qi. 2006. A Study on Ecosystem Health Assessment in YinChuan City. Journal of Xianyang Normal University04: 41-44.

[13] Yang Wei-fei, Li Tie-song, Pan An, Luo Jiang-ping. 2006. Assessment of Urban Ecosystem Health in Nanchong. Environmental Science and Management06:187-190.

[14] Yan Wen-tao, Yuan Xing-zhong, Xing Zhong. 2007. Urban Ecosystem Health Assessment Based on Attribute Theory: A Case Study in the New District of Northern Chongqing City. Chinese Journal of Ecology26(10): 1679-1684.

[15] Yu Jian-su. 2010. Health Assessment of Urban Ecosystems Based on a Projection Pursuit Model in Main Urban of West Coast Economic Zone. Journal of Jiangxi Agricultural University(Social Sciences Edition)09(3): 101-105.

[16] Yu Ya-Juan, Guo Huai-Cheng, Liu Yong. 2008. Syndromic City Illnesses Diagnosis and Urban Ecosystem Health Assessment. Acta Ecologica Sinica28(4): 1736-1747.

[17] Zhang Jun-hua, Yang Yao-hong, Chen Nan-xiang. 2010. Assessment for Urban Ecosystem Health Based on Fuzzy Clusting Method. Journal of Henan Agricultural University44(3): 60-364. 
[18] Zhang Mei-zhu, Qin Qu, Yang Hong. 2015. Application of attribute recognition model based on entropy coefficiency to the health assessment of urban ecosystem. Scientific and Technological Management of Land and Resources(1): 21-27.

[19] Zeng Yong, Shen Gen-xiang, Huang Shen-fa, Wang Min. 2005. Health Assessment of Urban Ecosystem in Shanghai. Resources and Environment in the Yangtze Basin14(2):208-212.

[20] Zheng Ying-hua, Li Sen, Li Zhi-lun. 2006. Assessment of Urban Ecosystem Health in Foshan. Journal of Foshan University(Natural Science Edition)01:56-58. 